Proceedings of the WELCOME Scientific Meeting on Hybrid Nanostructures, Toruń, Poland, August 28-31, 2011

\title{
Gold Nanoparticles with Elongated Shapes: Synthesis and Optical Properties
}

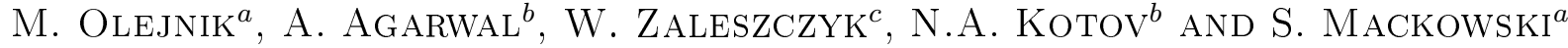 \\ ${ }^{a}$ Institute of Physics, Nicolaus Copernicus University, Grudziądzka 5, 87-100 Toruń, Poland \\ ${ }^{b}$ Department of Chemical Engineering, University of Michigan, Ann Arbor, Michigan 48109, USA \\ ${ }^{c}$ Institute of Physics, Polish Academy of Sciences, al. Lotników 32/46, 02-668 Warsaw, Poland
}

\begin{abstract}
We use a seed-mediated growth to fabricate gold nanorods in water solution. By changing the amount of silver salt we demonstrate the control of aspect ratio of the obtained rods. The microscopic and spectroscopic analyses provide ways to characterize the morphology and the optical properties of the gold nanorods. Nanorods synthesized using this approach feature length of the order of 50-60 nm, which translates into longitudinal plasmon resonances in the near infrared spectrum region.
\end{abstract}

PACS: 81.07.-b, 81.07.Pr, 87.64.kv, 87.64.K-

\section{Introduction}

Controlling plasmon excitation in nanoscale metallic particles has become important both from aspect of basic science as well as possible applications [1]. One of the outstanding challenges is to fabricate on-demand nanostructures with particular structural and optical properties. This includes shifting of plasmon resonance into the infrared spectral region, which is in the principle beyond reach for spherical nanoparticles [2]. Gold spherical nanoparticles, embedded in water environment $(n=$ 1.33 ), exhibit a surface plasmon resonance typically at $520 \mathrm{~nm}$ [3]. Through changing the size of a nanoparticle, we can shift the position of plasmon resonance up to $775 \mathrm{~nm}[2]$.

Recently it has been shown that plasmon resonance in silver island film can significantly enhance absorption of light-harvesting photosynthetic complexes in blue-green spectral region [4]. On the other hand, there exist many photosynthetic complexes with absorption bands in the red and infrared range of the electromagnetic spectrum. Among these, Photosystem I and Photosystem II are characterized with dominant absorption bands in red spectral region around $700 \mathrm{~nm}$ and $680 \mathrm{~nm}$, respectively, while light-harvesting complex 2 (LH2) from purple bacteria features two strong absorption bands in infrared: $800 \mathrm{~nm}$ and $850 \mathrm{~nm} \mathrm{[5].} \mathrm{The} \mathrm{optical} \mathrm{properties} \mathrm{of} \mathrm{these}$ complexes have been studied in detail during the last decades $[4,6,7]$.

Among the ways to shift the plasmon resonance to the infrared are fabrication of metallic nanoshells [8] and nanorods (NRs) [9-11]. Since nanorods possess two symmetry axes, there are two spectrally distinguishable resonances in their absorption spectra: transverse resonance, perpendicular to the long axis, and longitudinal plasmon resonance, along the axis of the nanorods [12]. While the energy of the transverse mode is located in the green spectral region, the energy of the longitudi- nal one is shifted far to the red and the infrared regions. It also strongly depends upon the length of the NRs. Thus we can explore ways to use this tunability in order to match absorption bands of the above-mentioned light-harvesting and photosynthetic systems and change transfer rates as well as enhance the fluorescence and absorption yields $[13,14]$.

In this work we report on synthesis of gold nanorods with longitudinal resonance in the range of 750-850 $\mathrm{nm}$. Structural data reveals reasonable homogeneity of the obtained nanoparticles. We also observe aging of the nanorods, which results in their shortening, thus shifting of the corresponding plasmon resonances.

\section{Experimental section}

$\mathrm{HAuCl}_{4} \cdot 3 \mathrm{H}_{2} \mathrm{O}(99.9 \%), \mathrm{NaBH}_{4}(99 \%)$, L-ascorbic acid $(99+\%)$, hexadecyltrimethylammoniumbromide (CTAB) (99\%), and $\mathrm{AgNO}_{3}(99+\%)$ were purchased from Aldrich. All chemicals were used without further purification. Deionized water (Fluka) was used in all experiments.

\subsection{Preparation of $A u$ seeds}

CTAB solution $(4.7 \mathrm{ml}, 0.1 \mathrm{M})$ was mixed with $25 \mu \mathrm{l}$ of $0.05 \mathrm{M} \mathrm{HAuCl}_{4}$. To the stirred solution, $0.3 \mathrm{ml}$ of $0.01 \mathrm{M} \mathrm{NaBH}_{4}$ was added, which resulted in the formation of brownish yellow solution. Seeds solution was kept at room temperature until further used.

\subsection{Preparation of Au nanorods}

For the synthesis we use Au seeds prepared before. The "seed-mediated" method was developed by Jana et al. $[9,15]$; it is carried out in aqueous solution at atmospheric pressure and near room temperature. Scheme of the reaction is shown in Fig. 1. Appropriate quantities and molarities of CTAB $(150 \mathrm{ml}, 0.1 \mathrm{M}), \mathrm{HAuCl}_{4}$ $(1.5 \mathrm{ml}, 0.05 \mathrm{M})$, L-ascorbic acid $(1.2 \mathrm{ml}, 0.1 \mathrm{M}), 0.01 \mathrm{M}$ 
$\mathrm{AgNO}_{3}(1.6 \mathrm{ml}, 1.8 \mathrm{ml}, 2 \mathrm{ml})$ and seed $(360 \mu \mathrm{l})$ water solutions were added one by one in a flask, followed by a gentle mixing. Addition of ascorbic acid, as a mild reduction agent, triggered a mixture color change from dark yellow to colorless. After addition of the seed solution, the mixtures were put into water bath and kept at constant temperature of $28^{\circ} \mathrm{C}$ for $2 \mathrm{~h}$. Obtained products were separated from unreacted substrate and spherical particles by centrifugation at $9.000 \mathrm{rpm}$ for $60 \mathrm{~min}$. The supernatant was removed using a pipette and the precipitate was redissolved in pure water.

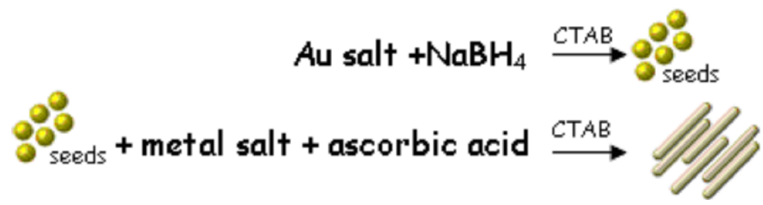

Fig. 1. Cartoon representation of gold nanorods synthesis through a "seed mediated" process.

The UV-visible spectra of the solutions were taken at room temperature, using quartz cuvette with a $1 \mathrm{~cm}$ optical path in the range 350-1100 $\mathrm{nm}$. Scanning electron microscope (SEM) studies were carried out with a Zeiss AURIGA microscope, operating at $5 \mathrm{kV}$, current $89 \mathrm{pA}$ and InLens detector. The samples of gold nanorods for SEM studies were diluted in pure water and then deposited on GaAs substrates as small droplets. All samples were allowed to dry in the ambient conditions. For every sample we determine the widths and lengths of approximately 50 nanoparticles for the sake of statistical analysis.

\section{Results and discussion}

The morphology of metallic nanoparticles was determined using SEM. In Fig. $2 \mathrm{a}-\mathrm{d}$ we show the images of gold nanorods synthesized with an addition of different amount of silver nitrate: $1.6,1.8$ and $2.0 \times 10^{-5} \mathrm{~mol}$, respectively. For all syntheses we observe large agglomerates of nanorods, but there are areas of the samples where individual nanorods can be found (a). The SEM images indicate that synthesis yields majority of rod-shaped particles form in aqueous surfactant solution. In addition, SEM data indicates existence of gold spheres in nanorods solution (less than 10\%).

In order to quantify the parameters of the nanorods we analyze lengths and widths of 50 individual nanoparticles. Figure 3 depicts the effect of adding $1.6 \times 10^{-5} \mathrm{~mol}$ (a) and $2 \times 10^{-5} \mathrm{~mol}$ (b) silver nitrate to identical growth solutions. The average length for both samples of nanorods was $55 \mathrm{~nm}$, while the width increases from $12 \mathrm{~nm}$ to $15 \mathrm{~nm}$ for sample (b) and (a), respectively. Consequently the average aspect ratio for nanorods obtained by adding $1.6 \times 10^{-5}$ mol silver nitrate was found to be 3.5 and for the NRs produced after adding $2 \times 10^{-5} \mathrm{~mol}$ it was 4.5 .

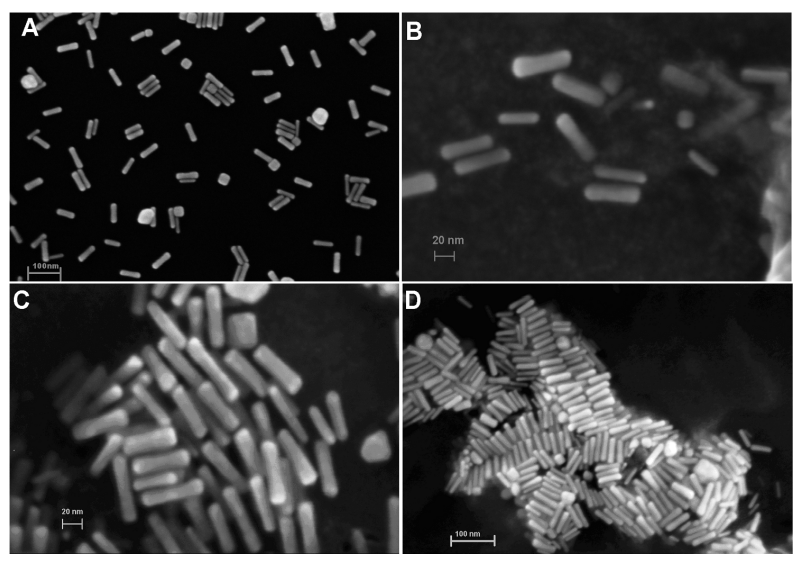

Fig. 2. SEM images of obtained products (A)-(D). The nanorod samples were prepared by an addition of identical amount of all chemicals with the exception of silver nitrate. The amount of $\mathrm{AgNO}_{3}$ was (A, B) $1.6 \times 10^{-5} \mathrm{~mol},(\mathrm{C}) 1.8 \times 10^{-5} \mathrm{~mol}$, and (D) $2.0 \times 10^{-5} \mathrm{~mol}$.

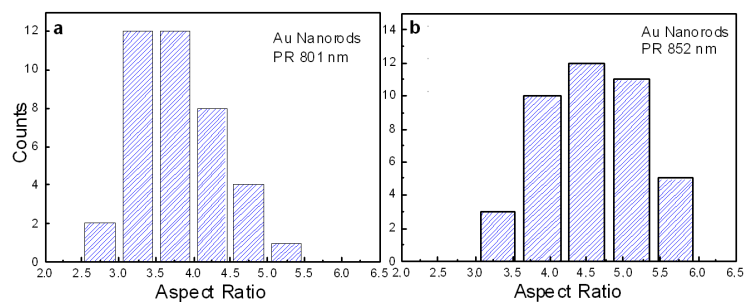

Fig. 3. Distribution of aspect ratio values for samples of gold nanorods synthesized with different amounts of silver nitrate: 1.6 (a) and $2 \times 10^{-5} \mathrm{~mol}(\mathrm{~b})$.

Spectroscopic methods such as UV-Vis spectroscopy provide information about energies of the plasmon resonances in metallic nanoparticles and to some degree about approximate size of product. This technique can also be used to monitor the reaction, as displayed in Fig. 4a. In the case of the nanorods the one distinct difference as compared to spherical particles is the appearance of two plasmon bands - the transverse and longitudinal ones. This leads to formation of two bands in absorption spectrum - a narrow peak around 570 and a broad band at wavelengths over $800 \mathrm{~nm}$. Also, a single peak due to spherical gold particles at $519 \mathrm{~nm}$ is expected during "seed-mediated" synthesis of nanorods.

Absorption spectra for nanorods growing from gold seeds as a function of time from 25 to 110 min after the addition of 5 min aged seeds to the growth solution are depicted in Fig. 4a. The spectra feature all three absorption bands, as described above. During the growth process the longitudinal plasmon resonance peak forms in the first $25 \mathrm{~min}$ and shifts from $788 \mathrm{~nm}$ through $821 \mathrm{~nm}$ to $801 \mathrm{~nm}$. At the same time the energy of the transverse plasmon resonance shifts monotonically from $561 \mathrm{~nm}$ to $579 \mathrm{~nm}$ (Fig. 4b). It suggests that the aspect ratio of the 


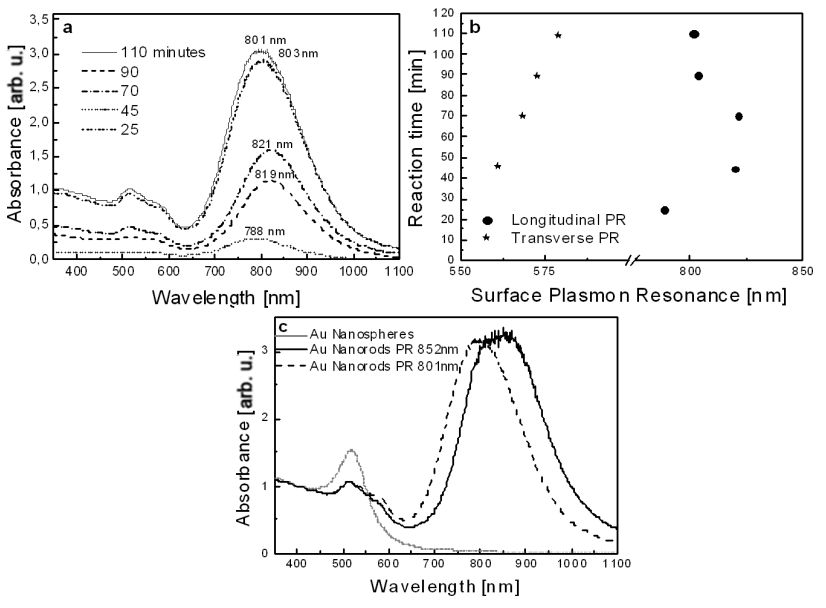

Fig. 4. (a) Absorption spectra of gold NR solution after seed addition taken at several times afterwards, as indicated; the longitudinal peak intensity increases with time; (b) variation of longitudinal and transverse plasmon band maxima as a function of time; (c) absorption spectra of two identical growth solutions, in which the silver content was changed from $1.6 \times 10^{-5}$ (dotted line) to $2 \times 10^{-5}$ (solid) mol. The spherical nanoparticle spectrum is also shown.

rods increases and then slowly decreases in time.

The position of a plasmon band in near-IR depends strongly on the silver nitrate amount used in synthesis. In Fig. 4c we compare the absorption spectra of spherical gold nanoparticles with the ones measured for nanorods synthesized with 1.6 and $2 \times 10^{-5} \mathrm{~mol}$ of the silver nitrate. It can be seen that with an increase of the silver concentration, the longitudinal plasmon band red-shifts from $801 \mathrm{~nm}$ to $852 \mathrm{~nm}$. Also, absorbance within this band increases relative to the transverse plasmon band. In all spectra we see contribution of gold spherical nanoparticles with the resonance at $519 \mathrm{~nm}$. The results shown in Fig. 4c indicate that we are able to control the length of the NRs by varying the silver ion content in the growth solution. Underpotential deposition (UPD) [15] mechanism explains the influence of the silver concentration upon the nanorods dimension and aspect ratio. Gold NRs, grown using CTAB-protected gold seeds in the presence of $\mathrm{Ag}^{+}$, bear $\mathrm{Au}\{110\}$ faces on the sides of the rods and $\{100\}$ faces at the ends. Increase of the concentration of silver substrate should result in more silver on $\mathrm{Au}\{100\}$ faces and the growth of nanorods with larger aspect ratios.

The results shown in Fig. 4 demonstrate that gold NRs are indeed suitable for studying plasmonic effects on light-harvesting complexes, as they match appropriate absorption bands thereof. One of the things that can limit the application of gold NRs to improve the absorption of light-harvesting complexes is their stability in water solution. In order to investigate the stability of gold nanorods synthesized in this work, the solution was kept at ambient condition for several months and its ab- sorption spectrum was measured in one month interval.

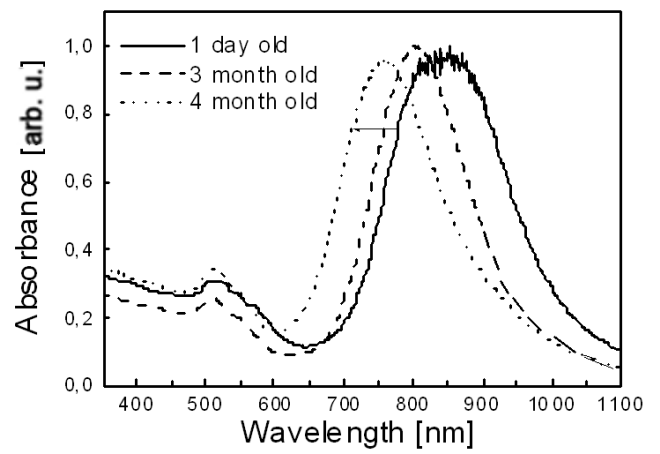

Fig. 5. Absorption spectra of gold nanorods taken at given time intervals after the synthesis.

Figure 5 shows the data obtained one day, 3 months and the 4 months after the nanorods were synthesized. We can see that the longitudinal plasmon resonance peak is narrowing and shifts towards the shorter wavelength range from 852 to $757 \mathrm{~nm}$. There is no significant change either in the transverse resonance peak, or the one resulting from the presence of spherical nanoparticles. We conclude that the nanorods are shrinking with time. This aging effect since it affects longitudinal plasmon energy is quite important and has to be considered before applying gold nanorods for attaching them to light-harvesting studies.

\section{Conclusions}

In conclusion, we have obtained rod-shaped nanoparticles with good reproducibility and yield. Obtained nanoparticles were characterized using scanning electron microscopy. The nanorods have lengths of about 50$60 \mathrm{~nm}$. The aspect ratio of the nanorods and thus the position of longitudinal plasmon resonance can be changed through adjusting the content of silver nitrate added during the reaction. We find the nanorods change their sizes with time; this effect should be taken into account when considering coupling the gold nanorods to light-harvesting complexes.

\section{Acknowledgments}

Financial support from the WELCOME program "Hybrid nanostructures as a stepping-stone towards efficient artificial photosynthesis" awarded by the Foundation for Polish Science is gratefully acknowledged. The research was partially supported by the European Union within European Regional Development Fund, through grant Innovate Economy (POIG.01.01.02-00-008/08). 


\section{References}

[1] H.A. Atwater, A. Polman, Nature Mater. 9, 205 (2010).

[2] C. Sönnichsen, T. Franzl, T. Wilk, G. von Plessen, J. Feldman, New J. Phys. 4, 93.1 (2002).

[3] C.J. Murphy, A.M. Gole, S.E. Hunyadi, W. John, P.N. Stone, Sisco, A. Alkilany, B.E. Kinarad, P. Hankins, Chem. Commun. 5, 544 (2008).

[4] N. Czechowski, P. Nyga, M.K. Schmidt, T.H.P. Brotosudarmo, H. Scheer, D. Piatkowski, S. Mackowski, Plasmonics 7, 115 (2012).

[5] R.E. Blankenship, Molecular Mechanism of Photosynthesis, MPG Books Ltd., Bodmin, Cornwall 2002.

[6] A.O. Govorov, I. Carmeli, Nano Lett. 7, 620 (2007).

[7] I. Carmeli, I. Lieberman, L. Kraversky, Z. Fan, A.O. Govorov, G. Markovich, S. Richter, Nano Lett. 10, 2069 (2010).

[8] R.D. Averitt, D. Sarkar, N.J. Halas, Phys. Rev. Lett. 78, 4217 (1997).
[9] C.J. Murphy, N.R. Jana, Adv. Mater. 14, 80 (2002).

[10] G.W. Bryant, F.J. Gararcia de Abajo, J. Aizpurua, Nano Lett. 8, 631 (2008).

[11] T.K. Sau, C.J. Murphy, Langmuir 20, 6414 (2004).

[12] M. Tréguer-Delapierre, J. Majimel, S. Mornet, E. Duguet, S. Ravaine, Gold Bull. 41, 195 (2008).

[13] S. Mackowski, J. Phys., Condens. Matter 22, 193102 (2010).

[14] Ł. Bujak, N. Czechowski, D. Piatkowski, R. Litvin, S. Mackowski, T.H.P. Brotosudarmo, R.J. Cogdell, S. Pichler, W. Heiss, Appl. Phys. Lett. 99, 173701 (2011).

[15] N.R. Jana, L. Gearheart, C.J. Murphy, Chem. Commun. 7, 617 (2001). 